\title{
Sense Theory
}

(part 6)

Sense Diagrams

[P-S Standard]

Egger Mielberg

egger.mielberg@gmail.com

27.09.2020

\begin{abstract}
.
Simple and readable diagrams of a complex set of any kind would allow a million of connections between elements of that set to be formulated and understood clearly. Current diagrams provide the visual solution for the first four-five sets mostly, but it is unreadable for the number of sets in more than five.

We propose a solution, sense diagrams, for visualization of multimillion sets using sense-to-sense paradigm [1]. The nature of the set can be any. The nature of the elements of a set may differ from each other. The main criterion of the use of this diagrams is the presence of qualitative or/and quantitative properties of an element of the set.
\end{abstract}

\section{Introduction}

There is currently no solution for describing sense-to-sense relationships between different objects. In many sciences such as chemistry, biology, quantum physics and others, there are research objects that have constant specific properties that distinguish them from other ones. These properties can be called genetic. In addition to genetic properties, objects have properties that can be common with other objects of a different 
nature. The sense diagram we present here is about visualization of relationships between different objects by the common properties.

\section{Problem}

There is no mathematical graphical description of the semantic relationship between two and more different objects.

\section{Solution}

Diagrams that describe the semantic relations between the elements of the sense space $S_{S}$ [2] are called sense diagrams.

In a rough approximation, the sense diagram describing the semantic shell for a separate zero object ${ }_{A}$ is similar to the description of Bohr's model of a hydrogen-like atom.
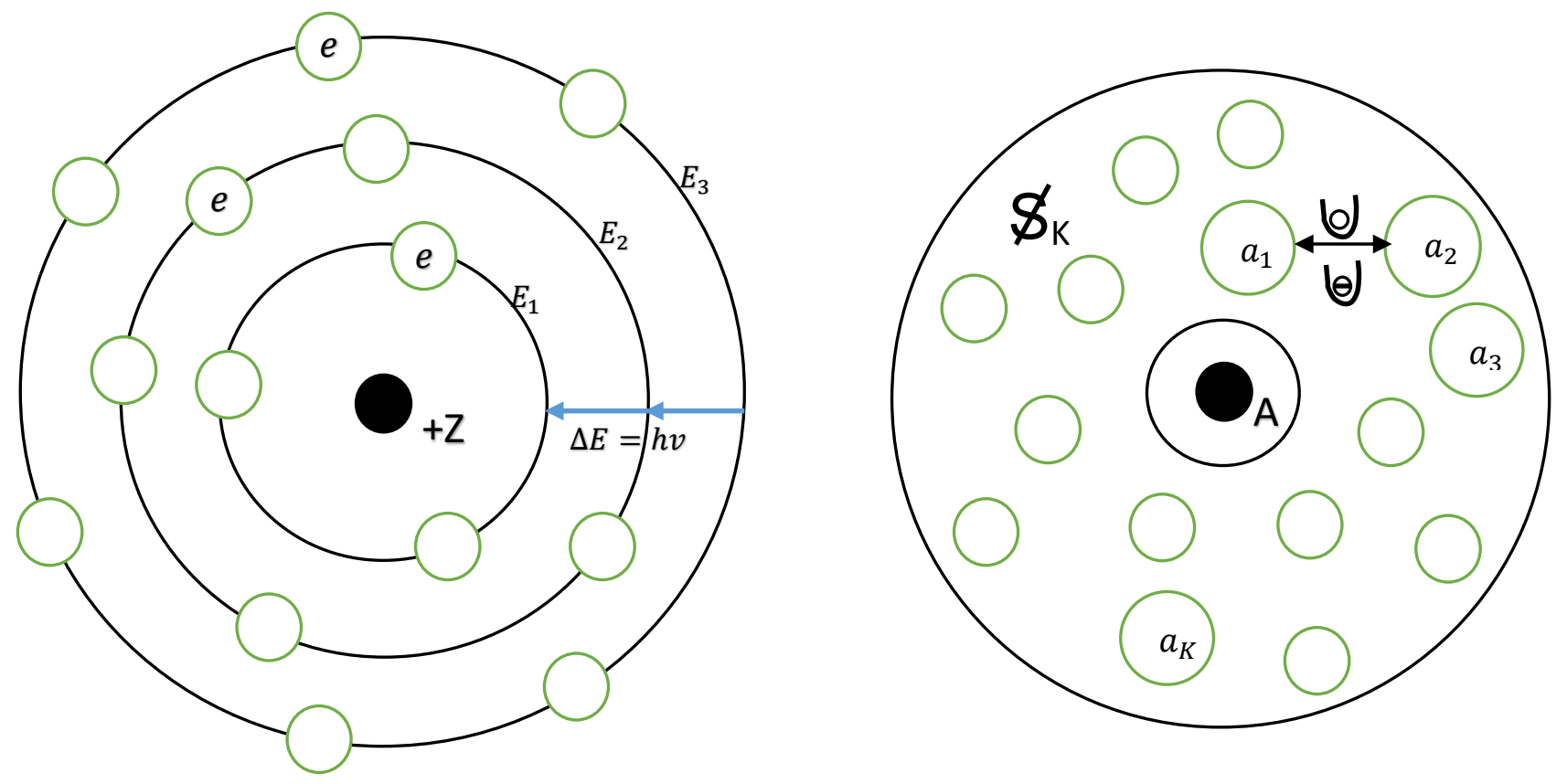

Pic. 1 
Similarity with Bohr's model of the atom:

1. Each element $a_{K}$ of the set ${ }^{\$_{k}}$ is associated with a certain zero object $O_{A(0)}$ just as each electron $e$ of each energy level $E_{n}$ is associated with a certain positively charged nucleus $+Z$.

2. Different elements $a_{1}, a_{2}, a_{3}, \ldots a_{K}$ of the set ${ }^{\$_{k}}$ have different values of the weights $\mathrm{W}_{S} i$ of their semantic connections with the zero object $O_{A(0)}$ just as all the electrons of one atom are at different energy levels $E_{i}$.

3. The sense set $S$ can contain many zero objects $\{\bigcirc\}_{n}$ described by one set ${ }^{\mathscr{S}_{N}}$ just as the nucleus of an atom contains several protons and neutrons.

4. For any No-Sense Set with the number of elements more than two tending to a certain zero object $O_{0}$, one can find a subset with the maximum values of the weights of semantic connections through the sense derivative on disunion [3] just as for the transition of an electron to a closer energy level to an atom it must give up a quantum of energy.

Differences from Bohr's model of the atom:

1. Each element of the sense series $A_{i}$ [4] can be associated in parallel with more than one zero object $O_{0}$, while a separate electron is associated with only one atom at a certain point in time. 
2. The set to which the sense series tends can contain only zero objects (protons), in contrast to the atomic nuclei of many chemical elements of the periodic table.

3. The elements of the sense series (zero object) can also be objects of different nature $O_{N(0)}$ or ${ }^{\$_{\text {o(N) }}}$, in contrast to the atomic nuclei of any chemical element where only electrons are at the energy levels.

4. The R-order semantic shell [2] can contain the number of elements of the sense space equal to or more than $2 N^{2}$.

5. In the Sense Theory, the connection between the zero object and No-Sense Set (Object No-Sense Set) is semantic, in Bohr's theory of the atom, the relationship between the nucleus and electrons is metric.

6. The Coherent Sense Set contains elements obtained from two or more simple elements of an arbitrary No-Sense Set (Object No-Sense Set). This situation is impossible for the electron shells of atoms of any chemical elements.

7. The level of semantic connection of the elements of the set ${ }^{\$_{N}}$ with the zero object $O_{0}$ does not depend on the order of the semantic shell, in contrast to the energetic connection of electrons with the nucleus located at different energy levels. 
Consider sense diagrams for the three main elements of the sense space, $O_{A(N)}, \quad \$_{N}, \$_{\text {o(N) }}$.

For $O_{A(N)}$

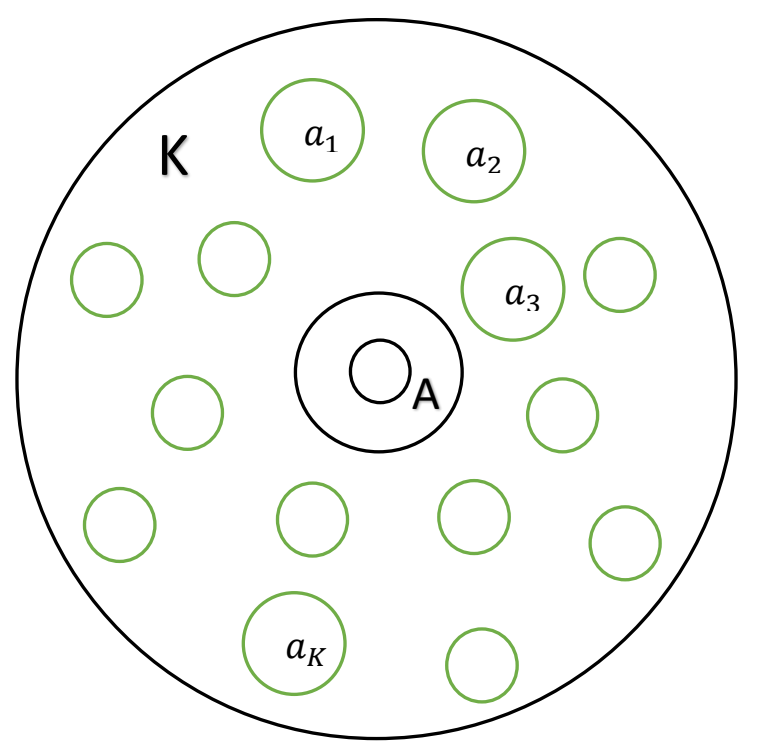

Pic. 2

For ${ }^{\$_{N}}$

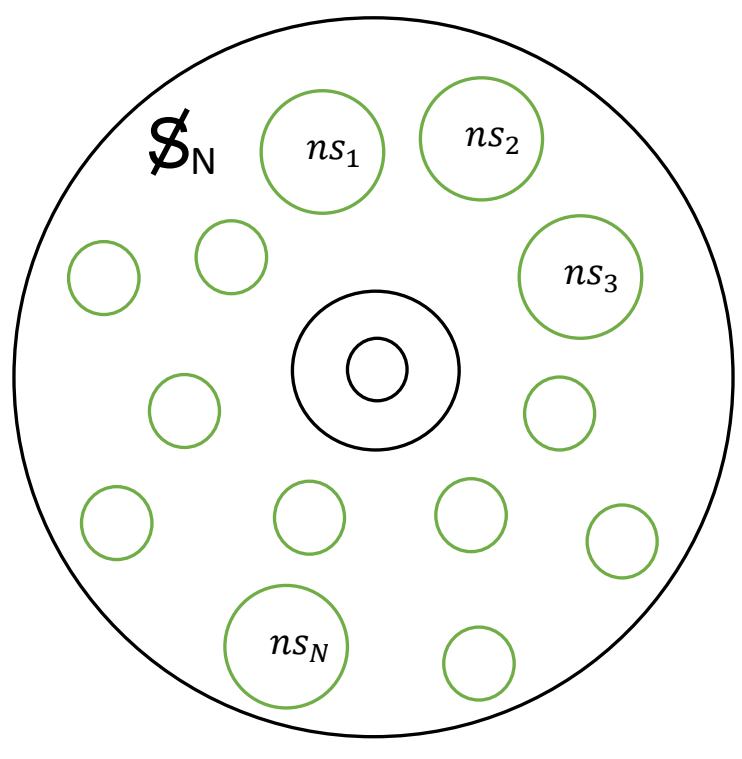

Pic. 3 
For ${ }^{\infty}{ }$

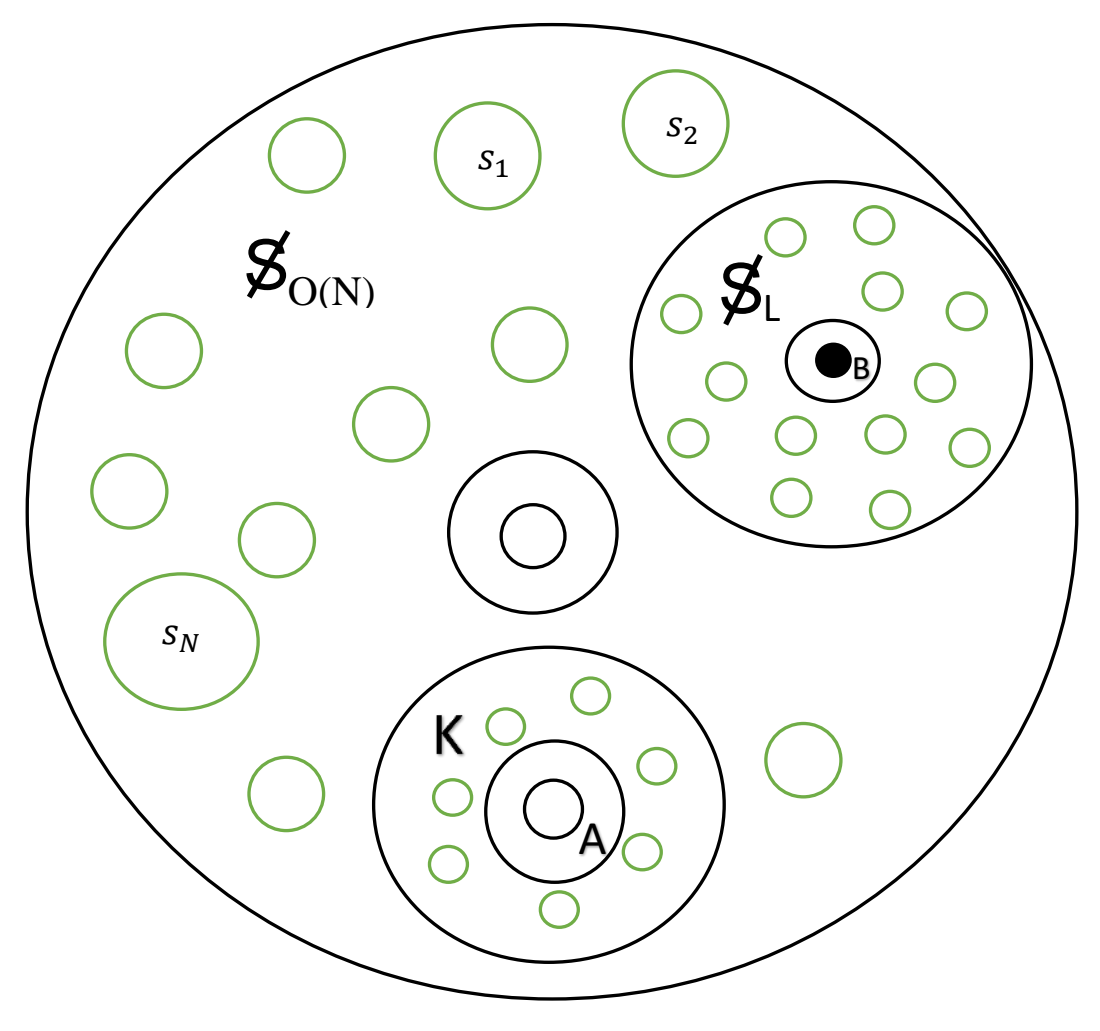

Pic. 4 


\section{For}

$$
O_{K} \text { b) } S_{\llcorner} \bullet S_{\mathrm{o}(\mathrm{M})}
$$

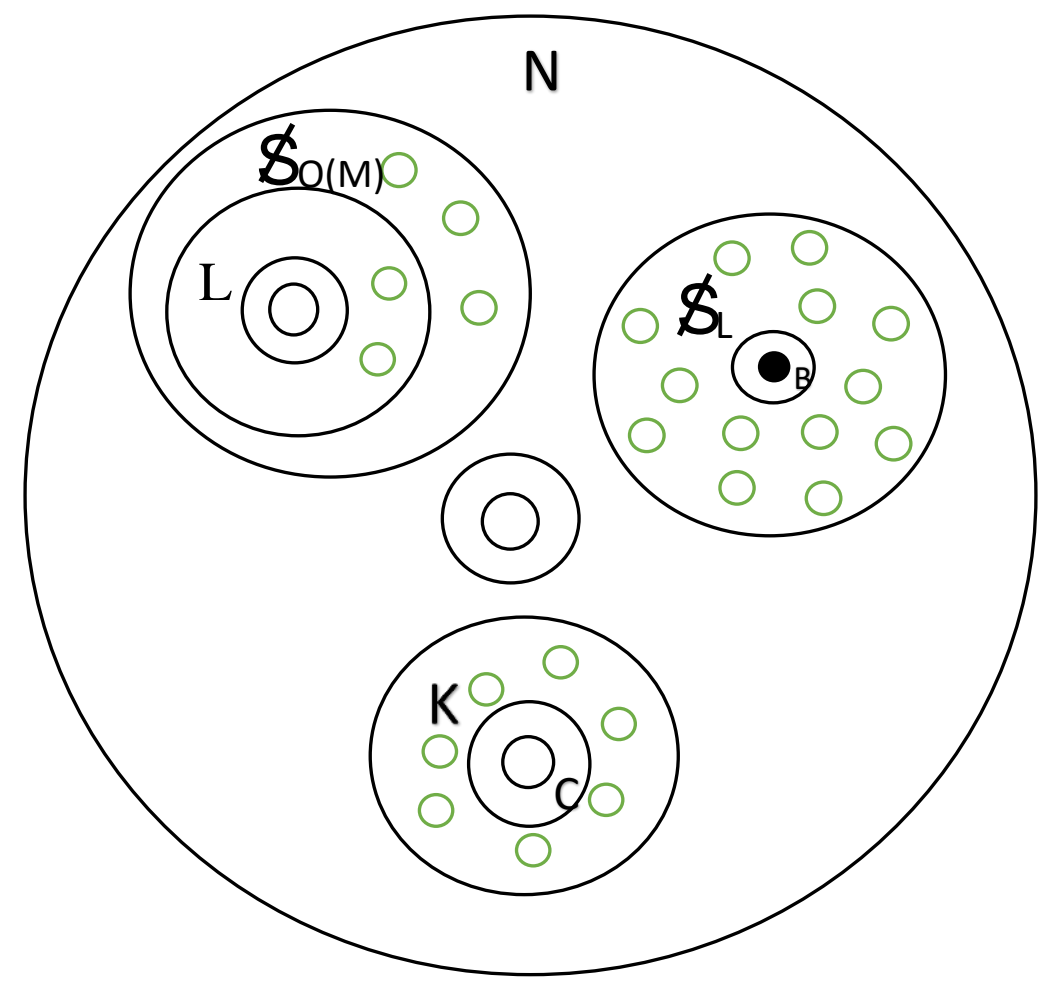

Pic. 5

$N=3$.

$\left\{O_{K}\right\}_{i}$ b) $\left\{\boldsymbol{S}_{\cup}\right\}_{i}$ b $\left\{\boldsymbol{S}_{\mathrm{o}(\mathrm{N})}\right\}_{i}$

For

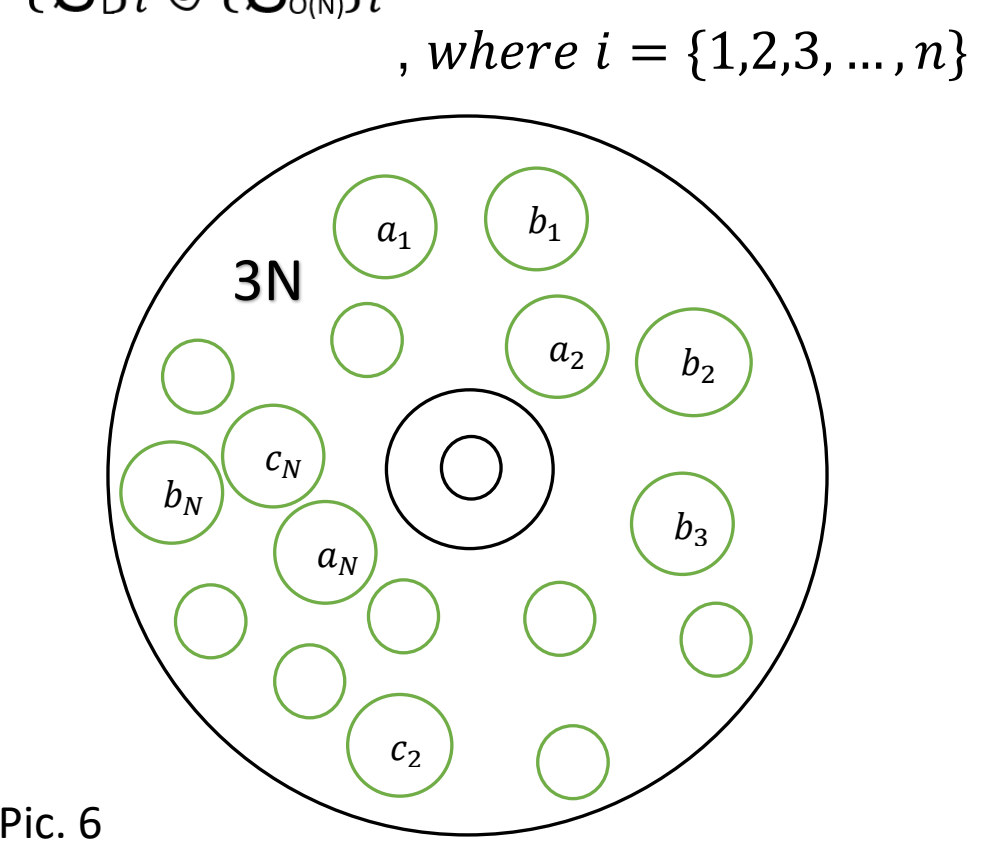


$\left\{a_{j}\right\}_{n} \in\left\{O_{K}\right\}_{i},\left\{b_{j}\right\}_{n} \in\left\{\mathscr{S}_{1}\right\}_{i},\left\{c_{j}\right\}_{n} \in\left\{\mathscr{S}_{\mathrm{o}(\mathrm{N})}\right\}_{i}$

For an infinitely large number of elements, sense diagrams are formed according to the principle of the induction method.

Sense diagrams describing the possible numerous

relationships between the elements of the sense space are also indicated in work [2].

Diagram for sense set $S_{A(K)}$ and object $O_{L}$ (in case of the existence of a sense derivative on object $A$ on union):

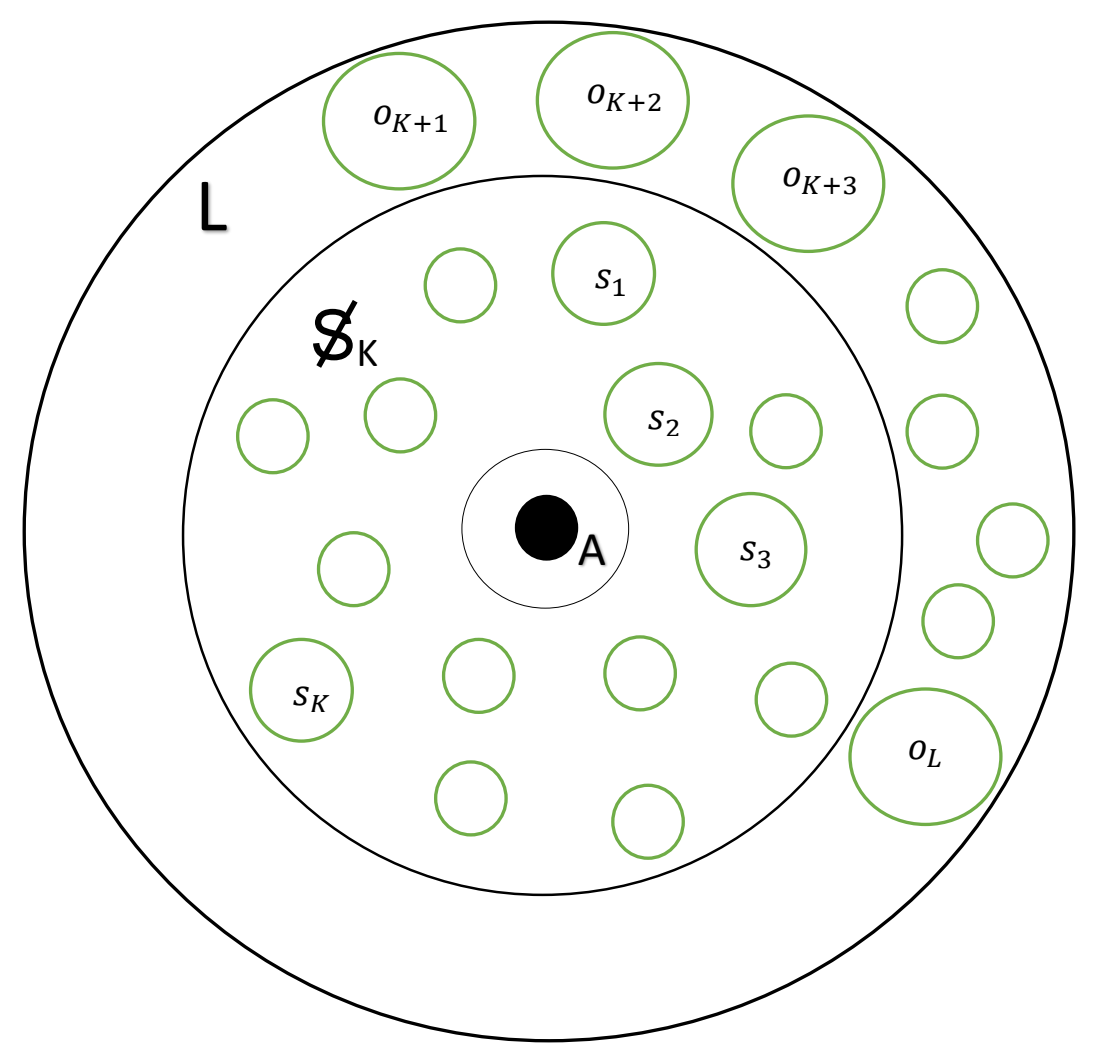

Pic. 7

Diagram for sense set $S_{A(K)}$, object $O_{L}$ and $\phi_{m}$ (in case of the existence of a sense derivative on object $A$ on union): 


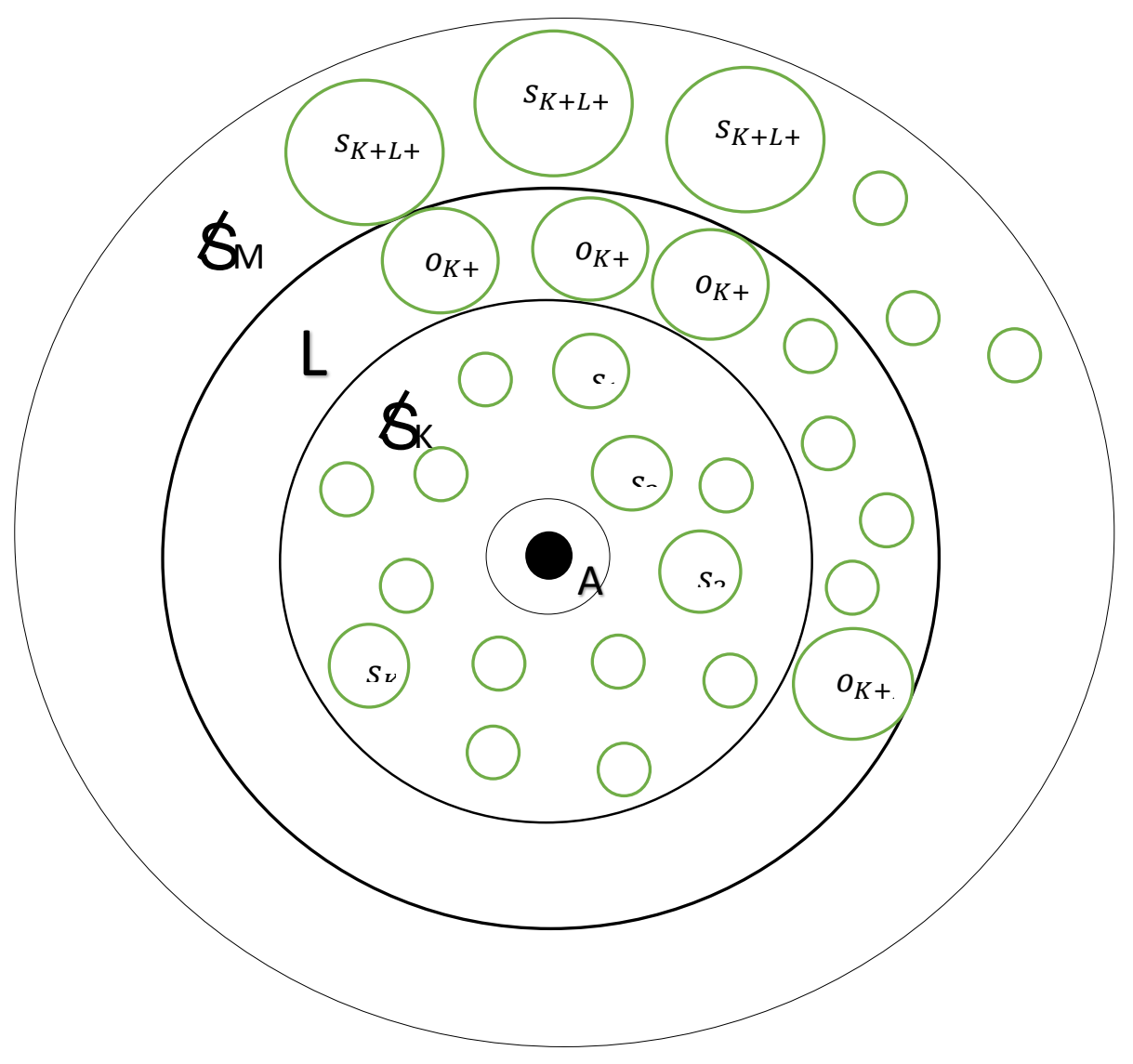

Pic. 8

A short notation of sets is also used.

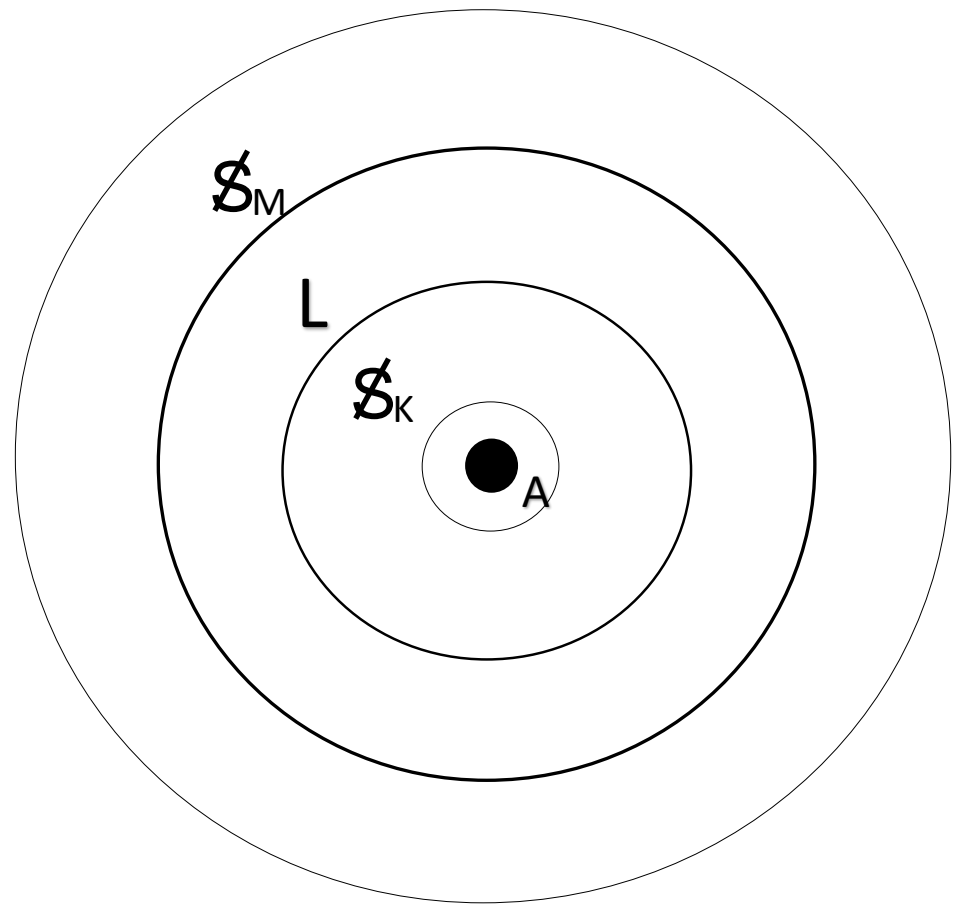

Pic. 9 
Diagram for two different sense sets $S_{A(K)}$ and $S_{B(L)}$ with the same elements $\delta_{k} \mid \underset{F=1}{E} s_{i}$ :

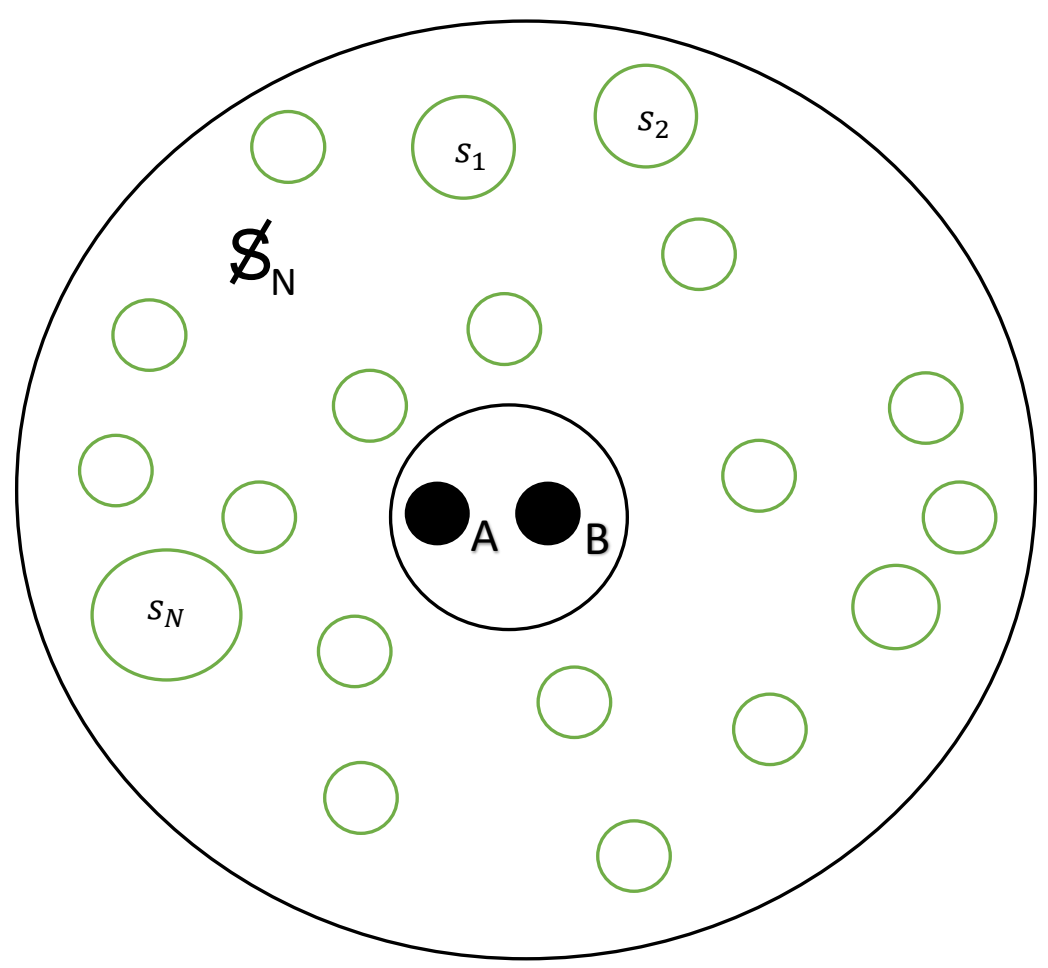

Pic. 10

$N=K$.

Elements of a sense diagram can be not only elements of a sense space, but also objects or events of a different nature.

\section{Conclusion}

In this article, we have presented a new type of diagram that allows a researcher to describe the semantic connections between trillions of objects and events of various nature. 
We hope that our decent work will help other Al researchers in their life endeavors.

To be continued. 


\section{References}

[1] E. Mielberg, "Sense Theory, Part 1", 2018, https://vixra.org/pdf/1905.0105v1.pdf

[2] E. Mielberg, "Sense Theory. Sense Space, Part 5", 2020, https://vixra.org/author/egger mielberg

[3] E. Mielberg, "Sense Theory. Derivative, Part 3", 2018, https://medium.com/@EggeMielbrerg/sense-theory-derivative-4d6fddd3b4f1

[4] E. Mielberg, "Sense Theory. Sense Series, Part 7", 2020, https://osf.io/6q5zp

[5] H. Kestler, A. Mueller, T. Gress, M. Buchholz, Generalized Venn diagrams: A new method of visualizing complex genetic set relations, 2005 ,

https://www.researchgate.net/publication/8156039 Generalized Venn diagr ams A new method of visualizing complex genetic set relations

[6] G. Waddell, R. Quinn, Two Applications of Venn Diagrams, 2011, https://www.researchgate.net/publication/264413124 Two Applications of Venn Diagrams

[7] G. Stapleton, P. Rodgers, J. Howse, J. Taylor, Properties of Euler Diagrams, 2007,

https://www.researchgate.net/publication/220053976 Properties of Euler D iagrams 